\title{
Choriocapillaris Degeneration in Geographic Atrophy
}

Elliott H. Sohn, ${ }^{* \dagger}$ Miles J. Flamme-Wiese, ${ }^{{ }^{\dagger}}$ S. Scott Whitmore, ${ }^{* \dagger}$ Grefachew Workalemahu, ${ }^{* \dagger}$ Alexander G. Marneros, ${ }^{\dagger}$ Erin A. Boese, ${ }^{* \dagger}$ Young H. Kwon, ${ }^{* \dagger}$ Kai Wang, ${ }^{* \top}$ Michael D. Abramoff, ${ }^{* \dagger}{ }^{* *}$ Budd A. Tucker, ${ }^{* \dagger}$ Edwin M. Stone, ${ }^{* \dagger}$ and Robert F. Mullins ${ }^{\star \dagger}$

From the Institute for Vision Research* and the Departments of Ophthalmology and Visual Sciences, ${ }^{\dagger}$ Biostatistics, ${ }^{\boldsymbol{\top}}$ Electrical and Computer Engineering, and Biomedical Engineering, ${ }^{* *}$ University of Iowa, Iowa City, Iowa; the Cutaneous Biology Research Center, ${ }^{\ddagger}$ Massachusetts General Hospital, Boston, Massachusetts; and the Department of Dermatology, ${ }^{\S}$ Harvard Medical School, Boston, Massachusetts

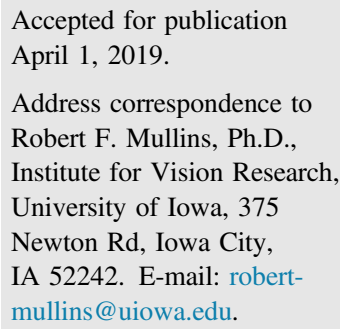

\begin{abstract}
Early age-related macular degeneration (AMD) is characterized by degeneration of the choriocapillaris, the vascular supply of retinal photoreceptor cells. We assessed vascular loss during disease progression in the choriocapillaris and larger vessels in the deeper choroid. Human donor maculae from controls $(n=99)$, early AMD $(n=35)$, or clinically diagnosed with geographic atrophy (GA; $n=9$, collected from outside the zone of retinal pigment epithelium degeneration) were evaluated using Ulex europaeus agglutinin-I labeling to discriminate between vessels with intact endothelial cells and ghost vessels. Morphometric analyses of choriocapillaris density (cross-sectional area of capillary lumens divided by length) and of vascular lumen/stroma ratio in the outer choroid were performed. Choriocapillaris loss was observed in early AMD (Bonferroni-corrected $P=0.024$ ) with greater loss in GA (Bonferronicorrected $P<10^{-9}$ ), even in areas of intact retinal pigment epithelium. In contrast, changes in lumen/ stroma ratio in the outer choroid were not found to differ between controls and AMD or GA eyes $(P>0.05)$, suggesting choriocapillaris changes are more prevalent in AMD than those in the outer choroid. In addition, vascular endothelial growth factor-A levels were negatively correlated with choriocapillaris vascular density. These findings support the concept that choroidal vascular degeneration, predominantly in the microvasculature, contributes to dry AMD progression. Addressing capillary loss in AMD remains an important translational target. (Am J Pathol 2019, 189: 1473-1480; https://doi.org/ 10.1016/j.ajpath.2019.04.005)
\end{abstract}

The choroid is a distinctive vascular tissue that supports vision by supplying oxygen and nutrients to the outer retina and retinal pigment epithelium (RPE) while removing waste. ${ }^{1,2}$ These functions are critical in light of the substantial metabolic activity of the neural retina. ${ }^{3}$ The choroid is a component of the peripheral circulatory system and has a high degree of blood flow, receiving $>80 \%$ of the ophthalmic artery's volume. ${ }^{4}$ The choroid has other unique features, including the following: i) an extremely dense capillary network (the choriocapillaris) with vascular lumens that occupy $>50 \%$ of the volume of the choriocapillaris layer in a healthy macula; ii) large-diameter capillaries that permit passage of multiple red blood cells; iii) diaphragm-bearing fenestrae, particularly on the inner (ie, facing the retina) surface; iv) a segmental pattern of supply and collection with anastomosing microvasculature; and v) a specific pattern of gene expression.

Although imaging the choroid in living subjects was formerly challenging, the development of the new imaging modalities, such as the enhanced depth imaging mode of spectral-domain optical coherence tomography (OCT) and OCT angiography (OCTA), has allowed the structure of the choroid to be evaluated in health and different disease states.

Supported, in part, by NIH grants EY024605 (R.F.M. and B.A.T.), EY026547 (E.H.S.), and P30 EY025580; the Elmer and Sylvia Sramek Charitable Foundation; and the Martin and Ruth Carver Chair in Ocular Cell Biology (R.F.M.).

Disclosures: None declared. 
The application of enhanced depth imaging mode of spectral-domain OCT has provided insight into the state of the choroid in several macular diseases, including central serous retinopathy, ${ }^{6}$ polypoidal vasculopathy, ${ }^{7}$ and high myopia. ${ }^{8}$ OCTA has enabled the noninvasive and rapid elucidation of the critical role the choriocapillaris plays in several vision-threatening diseases affecting the central retina, such as age-related macular degeneration (AMD),${ }^{9-11}$ acute macular neuroretinopathy, ${ }^{12}$ and diabetic retinopathy. ${ }^{13,14}$ The ability to quantify the structure of layers of the human macula using automated image analysis has made valuable contributions to our understanding of the dynamic nature of the retina and choroid in normal conditions and in response to various stimuli. ${ }^{15-19}$

The choroid has recently received renewed attention in the study of AMD, a common debilitating disease that affects millions of individuals in the United States alone. Although anti-vascular endothelial growth factor (VEGF) agents have been effective at limiting the vision loss associated with advanced, exudative, choroidal neovascularization, ${ }^{20-22}$ geographic atrophy (GA) remains a major cause of central vision loss. The central vision loss of GA is caused by the death of photoreceptor cells, RPE, and choriocapillary endothelial cells in the macular region of the retina. Before the widespread use of anti-VEGF agents, approximately $10 \%$ of the US population older than 70 years had GA, which was similar to the prevalence of exudative AMD. ${ }^{23}$ However, now that exudative AMD has an effective treatment that can restore vision and prevent vision loss, the fraction of patients with irreversible blindness from GA has increased. ${ }^{24}$ Moreover, there is some evidence that GA may be exacerbated in patients receiving anti-VEGF therapy. ${ }^{25}$

Although structural and functional changes in the choroid have classically been considered to be relatively late events in the pathogenesis of AMD (with RPE changes considered primary $^{26}$ ), several recent reports have described cellular and molecular changes in the choroid that occur early in disease. Functional studies of choroidal filling by Pauleikhoff et $\mathrm{al}^{27}$ and of subfoveal blood flow by Grunwald et $\mathrm{al}^{28}$ have shown slower filling and decreased choroidal volume and flow with progression of AMD and in association with abundance of drusen, early morphologic indicators of AMD. $^{29}$ Histologically, loss of viable choriocapillaris endothelium has been noted in human donor eyes in association with drusen ${ }^{30}$ before the gross dropout of RPE cells. ${ }^{5,31}$ Moreover, the choriocapillaris is the major site of deposition of the membrane attack complex of complement, which increases during normal aging and in AMD, suggesting a putative mechanism by which vascular injury could be linked to the AMD risk alleles of complement genes. ${ }^{32}$ These observations have led us and others to speculate that choriocapillaris degeneration may be a primary event in early AMD. Whether choroidal changes further contribute to the geographic atrophy of advanced dry AMD is less well understood.
For example, there has been some controversy about the relationship between vascular changes and geographic atrophy, with different results obtained using different approaches. ${ }^{31}$ McLeod et al ${ }^{33}$ studied whole mounts of human maculae and found that, although choriocapillaris vascular loss was notable in the center of atrophy, the edges had some preserved (although attenuated) vasculature with loss of RPE. Eyes with GA show choroidal thinning. ${ }^{34,35}$ In contrast, a recent report by Yiu et $\mathrm{al}^{36}$ found that (after correcting for refraction) GA is not associated with a thinner choroid. However, the choroidal thickness was related to the size of the atrophic lesion. Moreover, recent OCTA studies suggest that there is a concentric ring of vascular loss (or impaired flow) that is larger than the zone of RPE loss. ${ }^{9}$

Superimposed on the possible changes in choroidal thickness in GA, the choroid undergoes thinning during normal aging at the rate of approximately 1 to $2 \mu \mathrm{m}$ per year. ${ }^{37}$ The biological and anatomic basis of this thinning is not well understood, although it is accompanied by changes in the levels of protease inhibitors, with increased collagen and decreased ground substance. ${ }^{34}$ The relationship between the health of the choriocapillaris and choroidal thickness has not been exhaustively studied.

Our goal was to evaluate the relationship between the choriocapillaris, deeper choroidal vasculature, and choroidal thickness in unaffected eyes, eyes with early/intermediate AMD, and eyes clinically diagnosed with geographic atrophy. Morphologic features of the choroid were determined, and the vascular density of the choriocapillaris was decreased in eyes with AMD, and especially in GA, but the ratio of vascular lumen area/choroidal stroma area deeper than the choriocapillaris was not different between GA cases, AMD cases, and controls. Furthermore, the concentration of VEGF-A protein was negatively correlated with the density of the choriocapillaris in eyes without advanced AMD. These findings support the notion that choroidal vascular changes are notable in early AMD with functional consequences on VEGF-A levels that could predispose to advanced wet AMD.

\section{Materials and Methods}

\section{Human Donor Eyes}

Human donor eyes were selected from a large tissue collection of the Institute for Vision Research at the University of Iowa (Iowa City, IA). Eyes in this collection were obtained from the Iowa Lions Eye Bank after informed consent of the donor next of kin, and all experiments were performed in accordance with the Declaration of Helsinki. A total of 143 eyes were used, of which 35 had either a clinical diagnosis of AMD or were determined to have AMD by the histologic feature of confluent basal laminar deposit in the macula, ${ }^{38}$ as determined by one of us (R.F.M.) and 99 were unaffected controls. Eyes with a clinical diagnosis of geographic atrophy but with intact RPE in the studied 


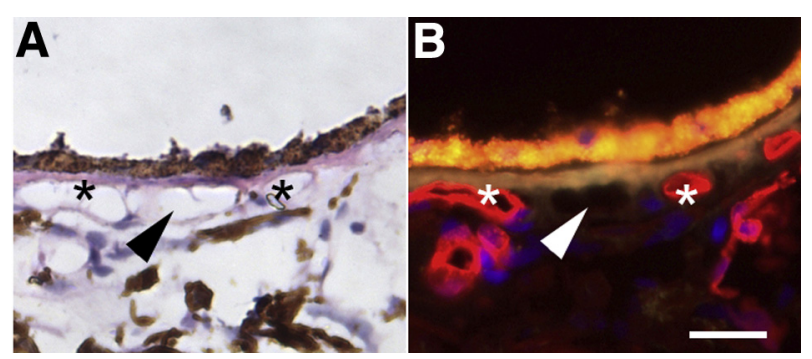

Figure 1 Hematoxylin-eosin-stained section (A) that was previously labeled with Ulex europaeus agglutinin-I (red; B). Section is from a 93year-old donor with early age-related macular degeneration. The presence of lumina with viable choriocapillaris endothelial cells (asterisks) and ghost vessels (arrowhead) are not readily distinguishable by hematoxylineosin staining. Scale bar $=25 \mu \mathrm{m}$.

sections $(n=9)$ were also included in the study. GA eyes sectioned through regions of RPE loss were excluded, and measurements were collected from within the macula, $>500$ $\mu \mathrm{m}$ from the central lesion. All samples used in the study were from donors aged 65 years and older. The average ages of donors in this study for controls, AMD, and GA were 80.0, 84.2, and 85.8 years, respectively. Eyes with choroidal neovascularization were not included in this data set, although in several cases the contralateral eyes had neovascular lesions. None of the donors examined in this study overlapped with a smaller, previous morphometric study on choriocapillaris. $^{30}$

\section{Histology}

Either whole or bisected macular punches ( $8 \mathrm{~mm}$ thick), centered on the fovea centralis, were fixed in $4 \%$ paraformaldehyde in phosphate-buffered saline for 2 hours, before cryoprotection in sucrose and embedding for cryostat sectioning, as described previously. ${ }^{30,39}$ Sections were labeled with biotin-conjugated Ulex europaeus agglutinin-I (UEA-I), which was visualized with avidin-Texas red (both from Vector Laboratories, Burlingame, CA), to detect endothelial cells of the choroid, as described previously. ${ }^{30}$ For some experiments, anti-CD45 antibody (BD Biosciences, San Jose, CA) was used in dual labeling, as described previously. ${ }^{30}$ One of the advantages of labeling the vasculature with UEA-I, in contrast to hematoxylineosin labeling, is that it allows the observer to discriminate between intact vessels lined with endothelial cells and ghost vessels, in which a vascular lumen is present but is not lined with endothelium (Figure 1).

\section{Morphometry}

Fluorescence micrographs from each donor were collected using a $20 \times$ objective and assembled using the photomerge tool in an image processing program (Adobe Photoshop CS version 8.0; Adobe, San Jose, CA). Files were then opened in ImageJ software version 1.52 (NIH, Bethesda, MD; http://imagej.nih.gov/ij), ${ }^{40}$ and pixels were converted to micrometers. Choriocapillaris vascular density $\left(\mu \mathrm{m}^{2} / \mu \mathrm{m}\right.$ length of Bruch membrane) was measured, as described previously. ${ }^{30}$ For choroidal thickness, measurements were collected from Bruch membrane to the innermost margin of sclera/outer aspect of the lamina fusca, as assessed by the regular pattern of fibrillar collagen. In addition, the total choroidal area was determined using the polygon tool, and the cross-sectional vascular areas were collected from all UEA-I-surrounded choroidal vessels, as depicted in Figure 2. In all cases, the investigator making the measurements was masked to the affection status of the donor. The unitless lumen/stroma ratio was calculated as the ratio of the nonchoriocapillaris vascular lumen area/the total area. Because three measurements were collected (thickness, choriocapillaris density, and lumen/stroma ratio), a Bonferroni correction factor of 3 was applied (ie, $P$ values were multiplied by 3 ).

In addition, the length of capillary/length of Bruch membrane ratio was measured in randomly selected fields from the 143 maculae used in this study, as described previously, ${ }^{46}$ except that UEA-I positivity was used to identify choriocapillaris vessels.

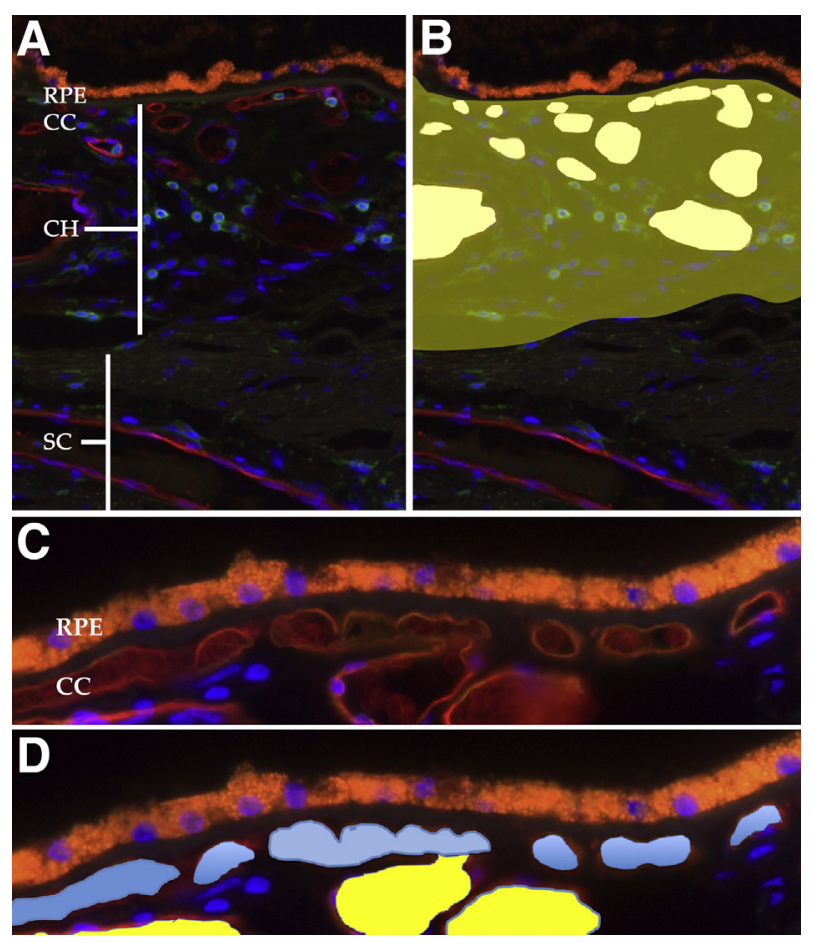

Figure 2 Examples of histologic sections used for quantification of choroidal features. A: Section labeled with Ulex europaeus agglutinin-I lectin (red) for vessel walls and antibodies directed against CD45 (green) for leukocytes, counterstained with DAPI (blue nuclei). The orange autofluorescence is caused by retinal pigment epithelium (RPE) lipofuscin. B: Cross-sectional areas of all vascular elements in the choroid $(\mathrm{CH})$ are quantified (white areas), and vascular areas are divided by total choroidal area (yellow shading) to determine the vascular lumen/stroma ratio. C: Highermagnification image of the choriocapillaris (CC) and deeper choroidal vessels. D: The segmentation of choriocapillaris (blue) versus larger vessels (yellow). Choriocapillaris lumen areas are measured and divided by length of Bruch membrane to derive the choriocapillaris density. SC, sclera. 


\section{VEGF Enzyme-Linked Immunosorbent Assay}

To measure the levels of VEGF-A, trephine punches $(6 \mathrm{~mm}$ thick), centered approximately $7 \mathrm{~mm}$ superonasal to the fovea, were collected from the neural retina of 14 donors and snap frozen within 8 hours of death. These juxtamacular punches were collected exactly adjacent to measured areas of choriocapillaris. None of the donors had advanced AMD (GA or CNV). Tissues were homogenized with disposable pestles (Sigma, St. Louis, MO) for 90 seconds in phosphatebuffered saline/1\% Triton X-100/protease inhibitor cocktail (Roche, Basel, Switzerland) buffer.

Protein concentration was determined using the Bradford assay reagents, according to the manufacturer's instructions (BioRad, Hercules, CA). VEGF-A was quantified in $20 \mu \mathrm{g}$ of retinal protein, analyzed in duplicate, using a human VEGF-A enzyme-linked immunosorbent assay kit, according to the manufacturer's instructions (Ray Biotech, Peachtree Corners, GA). Levels of VEGF-A protein were determined by linear interpolation based on a standard curve of 8.23 to $6000 \mathrm{pg} / \mathrm{mL}$ of VEGF-A.

\section{Results}

Vascular Loss in Early AMD and GA Is Predominant in the Choriocapillaris

Choriocapillaris vascular density was significantly higher in control eyes, compared with early AMD eyes (3.41 versus $2.61 \mu \mathrm{m}^{2} / \mu \mathrm{m} ; \quad P=0.009 ; \quad$ Bonferroni-corrected $P=0.027)$. This difference was especially remarkable when control eyes were compared with GA eyes $\left(1.33 \mu \mathrm{m}^{2} /\right.$ $\mu \mathrm{m}$; Bonferroni-corrected $P<10^{-6}$ ) (Figure $3 \mathrm{~A}$ and Table 1). In addition, GA eyes showed significant choriocapillaris dropout compared with early AMD (Bonferronicorrected $P<10^{-5}$ ) (Figure 3A). In contrast, the lumen/ stroma ratio (Figure 3B) (ie, vascular cross-sectional areas of large and intermediate vessels divided by total choroidal area) did not differ significantly between controls, early AMD, and GA donors (Table 1). Example images of the
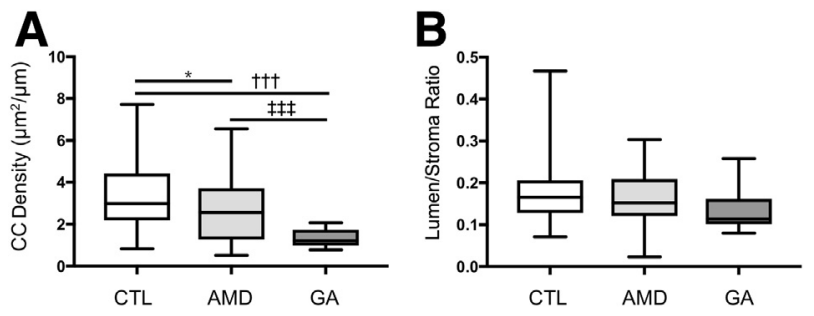

Figure 3 Morphometry of 143 donor maculae. A: Choriocapillaris (CC) vascular density $\left(\mu \mathrm{m}^{2} / \mu \mathrm{m}\right.$ length) is shown for control (CTL), early age-related macular degeneration (AMD), and geographic atrophy (GA) eyes. Choriocapillaris dropout is observed both in early AMD and GA eyes compared with control eyes. B: Choroidal vascular lumen area: stroma area across disease categories. ${ }^{*} P<0.05$ versus age-related macular degeneration; ${ }^{\dagger \dagger} P<0.001$ versus geographic atrophy; ${ }^{\ddagger \ddagger \ddagger} P<0.001$ versus geographic atrophy. choriocapillaris in age-matched control, early AMD, and GA eyes are depicted in Figure 4.

Similar results for capillary loss were obtained using the metric of capillary length/total length of Bruch membrane. This metric gave ratios in controls, early AMD, and GA maculae of $53.1 \%, 41.9 \%$, and $28.2 \%$, respectively. These values were significantly different between early AMD and controls $(P<0.001)$, GA and controls $\left(P<10^{-5}\right)$, and GA and early $\operatorname{AMD}(P<0.01)$.

\section{Choriocapillaris Density Is Not Related to Choroidal Thickness Measurements in Donor Eyes}

To determine whether choroidal thinning is related to choriocapillaris dropout, linear regression was performed using the 99 control donor maculae, in which choriocapillaris density and lumen/stroma ratios were analyzed on the basis of choroidal thickness (Figure 5). Data from these analyses show that there is only a weak relationship between choroidal thickness compared with choriocapillaris density $\left(r^{2}=0.01\right)$ and outer choroid lumen/stroma ratio $\left(r^{2}=0.04\right)$. Plots for total choroidal area versus vascular lumen area in controls, early AMD eyes, and GA eyes are depicted in Supplemental Figure S1. Relationships between choroidal thickness and vascular parameters for controls, early AMD, and GA maculae are depicted in Supplemental Figure S2.

\section{Eyes with Choroidal Vascular Loss Have Increased VEGF Levels in the Neural Retina}

To determine whether vascular loss contributes to retinal hypoxia, the amount of VEGF-A present in the neural retina of 14 eyes was determined. Levels of VEGF-A ranged from 38.5 to $442.3 \mathrm{pg} / \mathrm{mL}$. Regression analysis showed that vascular density was inversely related to VEGF-A levels $(P=0.015)$. In other words, retinas overlying atrophic choroids showed higher expression of VEGF-A than retinas overlying well-perfused choroids (Figure 6).

\section{Discussion}

Advances in imaging of the choroid, coupled with quantification using automated image analysis, have revealed that this unique tissue is dynamic, with measurable changes in thickness caused by environmental stimuli ${ }^{15,41}$ as well as changes in posture. ${ }^{42}$ Although choroidal thinning is a feature of AMD in many studies, the molecular mechanism of thinning - and whether it differs between normal aging and AMD-is not understood. We recently found that choroidal thinning is accompanied by a shift in protease inhibitors, with increased serine protease inhibitors in thick choroids and increased matrix metalloprotease inhibitors in thin choroids. This apparent shift was accompanied by an increased density of fibrillar collagen and loss of ground 
Table 1 Morphometric Features of the Choroid in Donor Maculae

\begin{tabular}{|c|c|c|c|c|c|}
\hline Measurement & Control group & AMD group & GA group & $\begin{array}{l}P_{\text {corr }} \text { for control vs } \\
\text { AMD group }\end{array}$ & $\begin{array}{l}P_{\text {corr }} \text { for control vs } \\
\text { GA group }\end{array}$ \\
\hline $\mathrm{CC}$ density, means $\pm \mathrm{SD}$ & $3.40 \pm 1.68$ & $2.61 \pm 1.5$ & $1.33 \pm 0.45$ & 0.028 & $<10^{-9}$ \\
\hline
\end{tabular}

Measurement data from controls, early/dry AMD, and GA donor maculae. $P$ values are corrected for multiple measurements.

AMD, age-related macular degeneration; $C C$, choriocapillaris; GA, geographic atrophy; $P_{\text {corr }}$ Bonferroni-corrected $P$ value.

substance in the choroidal stroma in thin choroids. ${ }^{34}$ In addition, we recently evaluated 100 donor eyes that were genotyped for a major risk factor in the $\mathrm{CFH}$ gene (rs1061170) and found that the choroid was significantly thinner in eyes with high-risk genotypes compared with those homozygous for the major, low-risk allele. ${ }^{32}$ Because $\mathrm{CFH}$ genotypes are also associated with levels of the complement membrane attack complex in the choroid, ${ }^{43}$ complement-mediated injury of the choriocapillaris is a plausible mechanism for the observed vascular dropout. ${ }^{5}$

In the current study, the overall lumen/stroma ratio did not differ between controls, AMD, and GA eyes. Also, the choriocapillaris did not show degeneration in thinner choroids (Figure 3). Choroidal thinning is observed in several conditions, most notably in high myopia. ${ }^{8}$ In an OCTA study of patients with high myopia, choroidal thinning was observed with increasing myopia and choriocapillaris flow voids (which may be areas of either no flow or low signal caused by flow velocity outside of the detection range of the instrument) appeared more often than in controls, but choroidal thickness changes were not correlated with these choriocapillaris flow voids. ${ }^{44}$ These findings parallel those of the current study that choriocapillaris density is largely independent of choroidal thickness. With choroidal alterations in both high myopia and AMD, one may wonder why patients with high myopia are not characterized by extensive drusen. The AMD risk alleles $C F H$ and ARMS2 are not highly associated with high myopia. ${ }^{45}$ Moreover, the findings in this study suggest that choriocapillaris degenerative changes are not related to thinning of the total choroid, such as that observed in high myopia. This loss of choriocapillaris density can take at least one of two forms. In some capillaries, the endothelium survives (on the basis of UEA labeling) but is narrowed, with an obliterated lumen. In other cases, the capillary endothelial cell is entirely missing, leaving behind an empty tube of extracellular matrix (or choriocapillaris ghost). Both of these patterns have been noted previously. ${ }^{33}$ In addition, under long-standing atrophy, ghost vessels appear to become replaced with extracellular matrix. Strategies for the regeneration of the diseased choriocapillaris will need to consider each of these stages.
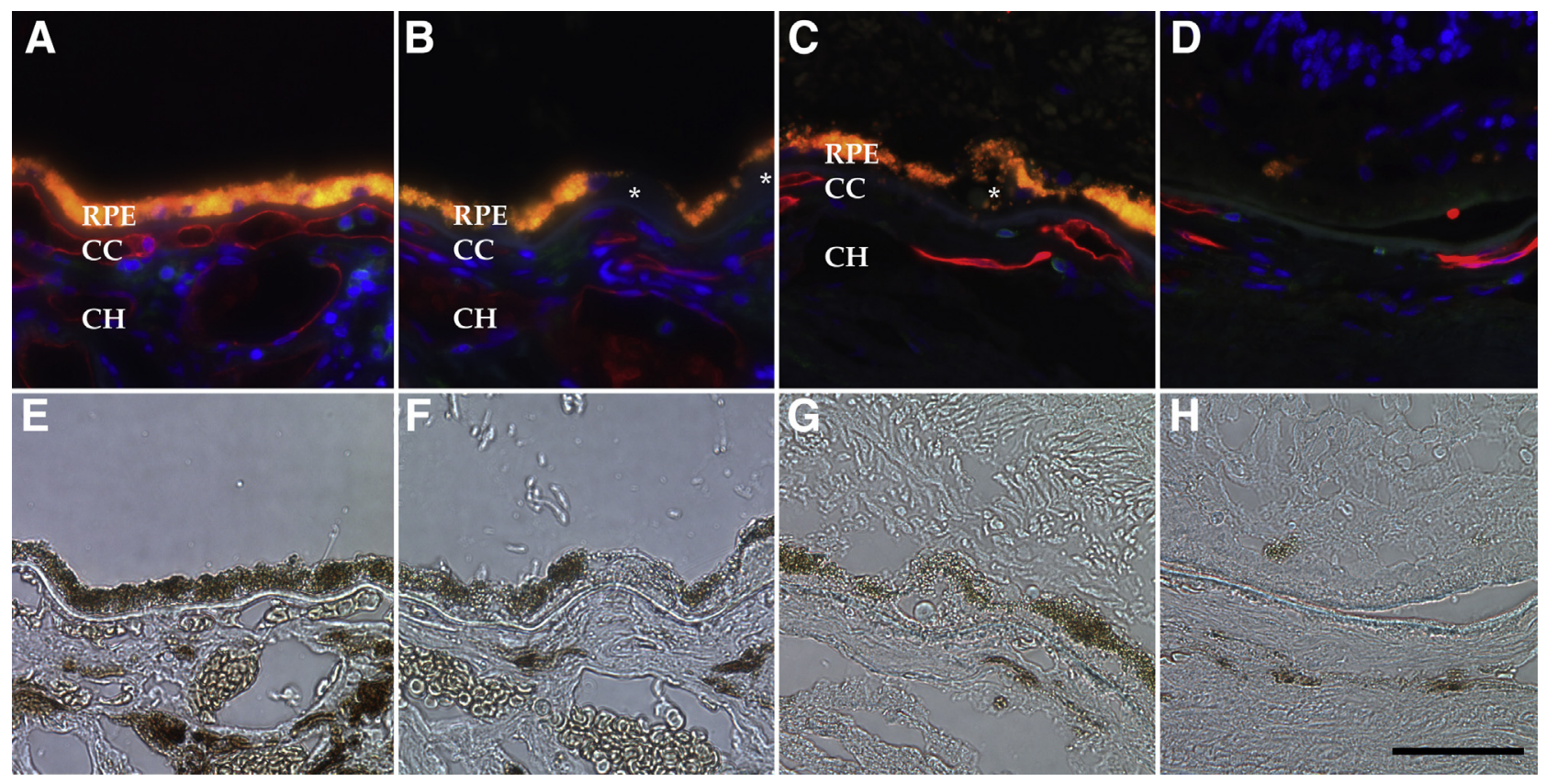

Figure 4 Examples of unaffected control (A and $\mathbf{E})$, early age-related macular degeneration (AMD; $\mathbf{B}$ and $\mathbf{F})$, and geographic atrophy (GA; C, D, G, and $\mathbf{H}$ ) maculae labeled with Ulex europaeus agglutinin-I (UEA-I) lectin (red) and anti-CD45 (green; A-D). Corresponding bright-field images are depicted in $\mathbf{E}$ through $\mathbf{H}$. Note the loss of UEA-I-positive capillaries over the course of AMD progression. $\mathbf{C}$ and $\mathbf{D}$ are from the same macula as $\mathbf{G}$ and $\mathbf{H}$, whereas $\mathbf{C}$ and $\mathbf{G}$ are outside of the central atrophic lesion depicted in $\mathbf{D}$ and $\mathbf{H}$. Donor ages are 86,82 , and 99 years, respectively. Asterisks indicate drusen. Scale bar $=50 \mu \mathrm{m}$. CC, choriocapillaris; $\mathrm{CH}$, outer choroid; RPE, retinal pigment epithelium. 

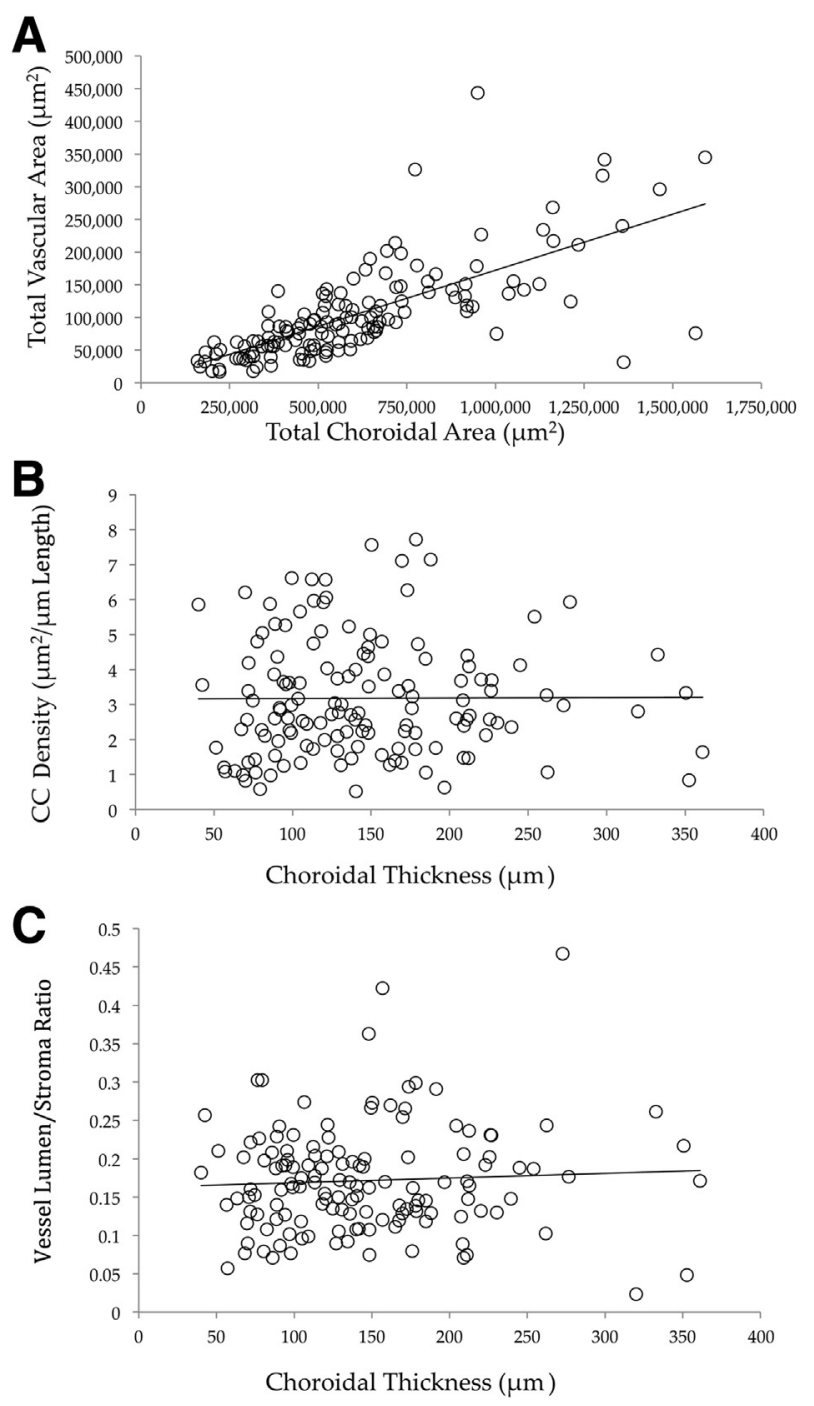

Figure 5 Vascular parameter relationship with choroidal thickness. A: Total vascular lumen area, plotted against total choroidal area, shows an expected relationship between these parameters. B and C: Choriocapillaris (CC) density $\left(\mu \mathrm{m}^{2} / \mu \mathrm{m}\right.$ length; B) and outer choroid (CH) lumen/stroma ratio $(C)$ were plotted against choroidal thickness $(\mu \mathrm{m})$. Neither $\mathrm{CC}$ nor $\mathrm{CH}$ vessel density is associated with choroidal thickness. Solid lines show regression lines.

A decrease in choriocapillaris density was noted in early AMD, with more striking loss in GA, and this loss was noted in sections with an intact RPE monolayer (ie, outside of the central zone of RPE atrophy). The AMD-associated loss of the choriocapillaris specifically has been reported previously using a variety of methods. ${ }^{30,31,46}$ Using wholemounted UEA-lectin-stained donor eyes, Seddon et al ${ }^{47}$ examined four controls, five GA eyes, as well as several eyes with early/intermediate AMD or choroidal neovascularization; and they showed choriocapillaris dropout without loss of RPE in neovascular AMD and found a 39\% loss of choriocapillaris in GA, with vascular narrowing but with better preservation outside of the central zone of RPE atrophy. Furthermore, gene expression ${ }^{48}$ and biochemical ${ }^{49}$ experiments on human AMD donor samples have generally supported the loss of endothelial cell-associated transcripts and proteins, respectively, with retention of RPE-associated molecules.

A recent histologic study evaluated a large series of wellcharacterized eyes with geographic atrophy using hematoxylin-eosin stain, and indicated photoreceptor cell dropout at the edges of atrophy. ${ }^{50}$ The choriocapillaris was reportedly normal under the atrophy. In contrast, a recent study using epoxy-embedded semithin sections found increased numbers of ghost capillaries adjacent to the GA lesions. ${ }^{51}$ One challenge in interpreting histologic data is that, because of the anatomic persistence of choriocapillaris ghost capillaries, hematoxylin-eosin-stained sections often overestimate the viability of the choriocapillaris (Figure 1). This is especially notable as nucleated leukocytes frequently migrate into the spaces formerly occupied by vascular channels, ${ }^{52}$ suggesting a greater degree of perfusion than is actually present (Figure 4). We propose that high-resolution, semithin sections, ${ }^{51}$ transmission electron microscopy, or robust, consistent markers of endothelial viability (eg, endogenous alkaline phosphatase activity ${ }^{53}$ or fucose-based UEA-I lectin histochemistry ${ }^{30}$ ) are necessary to definitively quantify the health and presence of the choroidal endothelium.

With increasing ability in the clinic to assess the state of the choriocapillaris, there is value in attempting to relate structural findings in this capillary bed to clinical measures. Different alterations in the choriocapillaris may have dissimilar impact on different imaging modalities. We observe normal capillaries, ghost vessels, and collapsed capillaries with highly constricted lumens (also noted at the edges of atrophy by McLeod et $\mathrm{al}^{33}$ ). OCTA ${ }^{9}$ requires passage of red blood cells over a fairly narrow range of velocities to observe flow, whereas fluorescein and indocyanine green are diffused in the plasma either free floating or bound to protein, respectively. ${ }^{27}$ Complete loss of the endothelium within a capillary segment, as observed in the many ghost capillaries in GA, is expected to result in a loss of signal from both OCTA and blood-borne dyes (because the

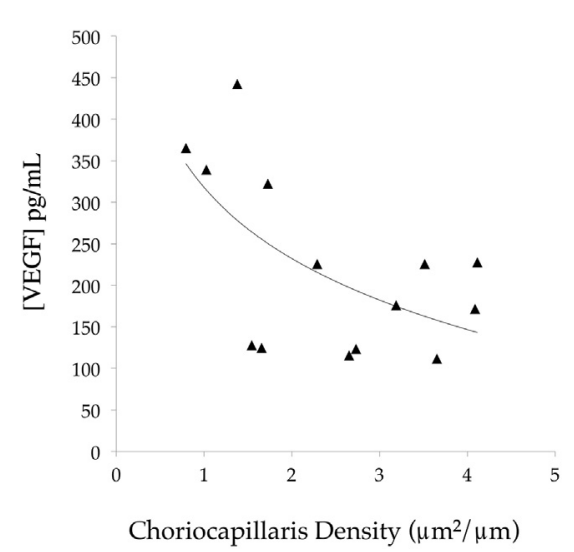

Figure 6 Relationship between choriocapillaris vascular density and retinal vascular endothelial growth factor (VEGF) concentration. In eyes with a low vascular density, VEGF levels are increased, consistent with retinal hypoxia overlying lost choroidal vasculature. Solid line shows trend line. 
endothelium is required to maintain the blood in a liquid state). On the other hand, the patent but extremely attenuated capillaries may not permit the passage of a detectable number of red blood cells, but they may allow passage of fluid, albeit at a much higher vascular resistance. Because resistance increases in proportion to the fourth power of the change in lumen radius, a relatively conservative change in diameter of $50 \%$ is expected in a 16 -fold increased vascular resistance. We speculate that the mixed population of attenuated and ghost vessels in eyes with GA outside of the central lesion (Figure 4) may result in the loss of signal from conventional OCTA and relatively decreased filling on fluorescein and indocyanine green angiography.

The potential consequences of choroidal degeneration on the neural retina and RPE are not fully understood, but possibilities include impaired clearance of lipid debris from RPE/Bruch membrane, loss of heat dissipation, and hypoxia. ${ }^{5}$ The possibility that a modest decrease in perfusion of the choriocapillaris could have a major impact on the health of the overlying retina is consistent with the findings of oxygen use in the mammalian retina, in which a high partial pressure of oxygen in the RPE and tips of the outer segment plunges to near 0 at the photoreceptor cell somas. ${ }^{54}$ The biochemical investigation of the neural retina in donor eyes found that VEGF-A levels were significantly and inversely associated with vascular density in adjacent choroid punches. This finding suggests that eyes exhibiting loss of choroidal vasculature may also undergo retinal hypoxia with consequential up-regulation of VEGF-A.

Taken together, these results suggest that the choroid degenerates in early AMD, and that this loss continues in advanced atrophic AMD, with molecular consequences in the overlying retinal cells. In light of the choroidal vascular changes that occur in this disease-in some cases in advance of RPE loss-we propose that treatment strategies for AMD will require consideration of the choroid at one or more stage of disease. In early AMD, therapeutic interventions that protect the choroidal vasculature from degeneration (from complement-mediated injury, loss of trophic support from the RPE, or other insults) are needed to prevent progression toward atrophy or choroidal neovascularization. In eyes that have already experienced GA, strategies to rebuild the choroid (eg, using patient-derived choroidal endothelial cells) ${ }^{55}$ may be required to fully repair the degenerated macula.

\section{Acknowledgments}

We thank the Iowa Lions Eye Bank and the eye donors and their families for the generous and crucial role in this research; and Christopher Barnard, Olivia Salter, and Brynnon Harman for technical assistance.

\section{Supplemental Data}

Supplemental material for this article can be found at http://doi.org/10.1016/j.ajpath.2019.04.005.

\section{References}

1. Nickla DL, Wallman J: The multifunctional choroid. Prog Retin Eye Res 2010, 29:144-168

2. Chirco KR, Sohn EH, Stone EM, Tucker BA, Mullins RF: Structural and molecular changes in the aging choroid: implications for agerelated macular degeneration. Eye (Lond) 2017, 31:10-25

3. Linsenmeier RA, Braun RD: Oxygen distribution and consumption in the cat retina during normoxia and hypoxemia. J Gen Physiol 1992, 99:177-197

4. Harris A: Atlas of Ocular Blood Flow. Philadelphia, PA: Butterworth Heinemann, 2003

5. Whitmore SS, Sohn EH, Chirco KR, Drack AV, Stone EM, Tucker BA, Mullins RF: Complement activation and choriocapillaris loss in early AMD: implications for pathophysiology and therapy. Prog Retin Eye Res 2014, 45:1-29

6. Imamura Y, Fujiwara T, Margolis R, Spaide RF: Enhanced depth imaging optical coherence tomography of the choroid in central serous chorioretinopathy. Retina 2009, 29:1469-1473

7. Peiretti E, Pozzoni MC, Fossarello M, Spaide RF: Polypoidal choroidal vasculopathy in association with choroidal nevus. Retin Cases Brief Rep 2009, 3:12-14

8. Fujiwara T, Imamura Y, Margolis R, Slakter JS, Spaide RF: Enhanced depth imaging optical coherence tomography of the choroid in highly myopic eyes. Am J Ophthalmol 2009, 148:445-450

9. Choi W, Moult EM, Waheed NK, Adhi M, Lee B, Lu CD, de Carlo TE, Jayaraman V, Rosenfeld PJ, Duker JS, Fujimoto JG: Ultrahigh-speed, swept-source optical coherence tomography angiography in nonexudative age-related macular degeneration with geographic atrophy. Ophthalmology 2015, 122:2532-2544

10. Jia Y, Bailey ST, Hwang TS, McClintic SM, Gao SS, Pennesi ME, Flaxel CJ, Lauer AK, Wilson DJ, Hornegger J, Fujimoto JG, Huang D: Quantitative optical coherence tomography angiography of vascular abnormalities in the living human eye. Proc Natl Acad Sci U S A 2015, 112:E2395-E2402

11. Roisman L, Zhang Q, Wang RK, Gregori G, Zhang A, Chen C-L, Durbin MK, An L, Stetson PF, Robbins G, Miller A, Zheng F, Rosenfeld PJ: Optical coherence tomography angiography of asymptomatic neovascularization in intermediate age-related macular degeneration. Ophthalmology 2016, 123:1309-1319

12. Lee SY, Cheng JL, Gehrs KM, Folk JC, Sohn EH, Russell SR, Guo Z, Abràmoff MD, Han IC: Choroidal features of acute macular neuroretinopathy via optical coherence tomography angiography and correlation with serial multimodal imaging. JAMA Ophthalmol 2017 , 135:1177-1183

13. Abràmoff $\mathrm{MD}$, Fort $\mathrm{PE}$, Han IC, Jayasundera KT, Sohn EH, Gardner TW: Approach for a clinically useful comprehensive classification of vascular and neural aspects of diabetic retinal disease. Invest Ophthalmol Vis Sci 2018, 59:519-527

14. Nesper PL, Roberts PK, Onishi AC, Chai H, Liu L, Jampol LM, Fawzi AA: Quantifying microvascular abnormalities with increasing severity of diabetic retinopathy using optical coherence tomography angiography. Invest Ophthalmol Vis Sci 2017, 58:BIO307-BIO315

15. Arora KS, Jefferys JL, Maul EA, Quigley HA: Choroidal thickness change after water drinking is greater in angle closure than in open angle eyes. Invest Ophthalmol Vis Sci 2012, 53:6393-6402

16. Abràmoff $\mathrm{MD}$, Mullins RF, Lee K, Hoffmann JM, Sonka M, Critser DB, Stasheff SF, Stone EM: Human photoreceptor outer segments shorten during light adaptation. Invest Ophthalmol Vis Sci 2013, 54:3721-3728

17. Vural AD, Kara N, Sayin N, Pirhan D, Ersan HBA: Choroida thickness changes after a single administration of coffee in healthy subjects. Retina 2014, 34:1223-1228

18. Kim DY, Fingler J, Zawadzki RJ, Park SS, Morse LS, Schwartz DM, Fraser SE, Werner JS: Optical imaging of the chorioretinal vasculature in the living human eye. Proc Natl Acad Sci U S A 2013, 27:14354-14359 
19. Kim DY, Silverman RH, Chan RVP, Khanifar AA, Rondeau M, Lloyd H, Schlegel P, Coleman DJ: Measurement of choroidal perfusion and thickness following systemic sildenafil (Viagra( $\left({ }^{\circledR}\right)$ ). Acta Ophthalmol 2013, 91:183-188

20. CATT Research Group, Martin DF, Maguire MG, Ying G-S, Grunwald JE, Fine SL, Jaffe GJ: Ranibizumab and bevacizumab for neovascular agerelated macular degeneration. N Engl J Med 2011, 364:1897-1908

21. Bakall B, Folk JC, Boldt HC, Sohn EH, Stone EM, Russell SR, Mahajan VB: Aflibercept therapy for exudative age-related macular degeneration resistant to bevacizumab and ranibizumab. Am J Ophthalmol 2013, 156:15-22.e1

22. Tozer K, Roller AB, Chong LP, Sadda S, Folk JC, Mahajan VB, Russell SR, Boldt HC, Sohn EH: Combination therapy for neovascular age-related macular degeneration refractory to anti-vascular endothelial growth factor agents. Ophthalmology 2013, 120: 2029-2034

23. Friedman DS, O'Colmain BJ, Muñoz B, Tomany SC, McCarty C, de Jong PTVM, Nemesure B, Mitchell P, Kempen J; Eye Diseases Prevalence Research Group: Prevalence of age-related macular degeneration in the United States. Arch Ophthalmol 2004, 122:564-572

24. Grunwald JE, Daniel E, Huang J, Ying G-S, Maguire MG, Toth CA, Jaffe GJ, Fine SL, Blodi B, Klein ML, Martin AA, Hagstrom SA, Martin DF; CATT Research Group: Risk of geographic atrophy in the comparison of age-related macular degeneration treatments trials. Ophthalmology 2014, 121:150-161

25. Grunwald JE, Pistilli M, Daniel E, Ying G-S, Pan W, Jaffe GJ, Toth CA, Hagstrom SA, Maguire MG, Martin DF: Comparison of age-related macular degeneration treatments trials research group: incidence and growth of geographic atrophy during 5 years of comparison of age-related macular degeneration treatments trials. Ophthalmology 2017, 124:97-104

26. Hogan MJ: Role of the retinal pigment epithelium in macular disease. Trans Am Acad Ophthalmol Otolaryngol 1972, 76:64-80

27. Pauleikhoff D, Spital G, Radermacher M, Brumm GA, Lommatzsch A, Bird AC: A fluorescein and indocyanine green angiographic study of choriocapillaris in age-related macular disease. Arch Ophthalmol 1999, 117:1353-1358

28. Grunwald JE, Metelitsina TI, Dupont JC, Ying G-S, Maguire MG: Reduced foveolar choroidal blood flow in eyes with increasing AMD severity. Invest Ophthalmol Vis Sci 2005, 46:1033-1038

29. Berenberg TL, Metelitsina TI, Madow B, Dai Y, Ying G-S, Dupont JC, Grunwald L, Brucker AJ, Grunwald JE: The association between drusen extent and foveolar choroidal blood flow in agerelated macular degeneration. Retina 2012, 32:25-31

30. Mullins RF, Johnson MN, Faidley EA, Skeie JM, Huang J: Choriocapillaris vascular dropout related to density of drusen in human eyes with early age-related macular degeneration. Invest Ophthalmol Vis Sci 2011, 52:1606-1612

31. Biesemeier A, Taubitz T, Julien S, Yoeruek E, Schraermeyer U: Choriocapillaris breakdown precedes retinal degeneration in agerelated macular degeneration. Neurobiol Aging 2014, 35:2562-2573

32. Mullins RF, Schoo DP, Sohn EH, Flamme-Wiese MJ, Workamelahu G, Johnston RM, Wang K, Tucker BA, Stone EM: The membrane attack complex in aging human choriocapillaris: relationship to macular degeneration and choroidal thinning. Am J Pathol 2014, 184:3142-3153

33. McLeod DS, Grebe R, Bhutto I, Merges C, Baba T, Lutty GA: Relationship between RPE and choriocapillaris in age-related macular degeneration. Invest Ophthalmol Vis Sci 2009, 50:4982-4991

34. Sohn EH, Khanna A, Tucker BA, Abràmoff MD, Stone EM, Mullins RF: Structural and biochemical analyses of choroidal thickness in human donor eyes. Invest Ophthalmol Vis Sci 2014, 55: $1352-1360$

35. Adhi M, Lau M, Liang MC, Waheed NK, Duker JS: Analysis of the thickness and vascular layers of the choroid in eyes with geographic atrophy using spectral-domain optical coherence tomography. Retina 2014, 34:306-312
36. Yiu G, Chiu SJ, Petrou PA, Stinnett S, Sarin N, Farsiu S, Chew EY, Wong WT, Toth CA: Relationship of central choroidal thickness with agerelated macular degeneration status. Am J Ophthalmol 2015, 159:617-626

37. Shin JW, Shin YU, Cho HY, Lee BR: Measurement of choroidal thickness in normal eyes using 3D OCT-1000 spectral domain optical coherence tomography. Korean J Ophthalmol 2012, 26:255-259

38. Sarks SH, Sarks SH: Ageing and degeneration in the macular region: a clinico-pathological study. Br J Ophthalmol 1976, 60:324-341

39. Barthel LK, Raymond PA: Improved method for obtaining 3-microns cryosections for immunocytochemistry. J Histochem Cytochem 1990, 38:1383-1388

40. Abramoff MD, Magalhães PJ, Ram SJ: Image processing with ImageJ. Biophotonics International 2004, 11:36-42

41. Vance SK, Imamura Y, Freund KB: The effects of sildenafil citrate on choroidal thickness as determined by enhanced depth imaging optical coherence tomography. Retina 2011, 31:332-335

42. Almeida DRP, Zhang L, Chin EK, Mullins RF, Kucukevcilioglu M, Critser DB, Sonka M, Stone EM, Folk JC, Abràmoff MD, Russell SR: Comparison of retinal and choriocapillaris thicknesses following sitting to supine transition in healthy individuals and patients with age-related macular degeneration. JAMA Ophthalmol 2015, 133:297-303

43. Mullins RF, Dewald AD, Streb LM, Wang K, Kuehn MH, Stone EM: Elevated membrane attack complex in human choroid with high risk complement factor H genotypes. Exp Eye Res 2011, 93:565-567

44. Al-Sheikh M, Phasukkijwatana N, Dolz-Marco R, Rahimi M, Iafe NA, Freund KB, Sadda SR, Sarraf D: Quantitative OCT angiography of the retinal microvasculature and the choriocapillaris in myopic eyes. Invest Ophthalmol Vis Sci 2017, 58:2063-2069

45. Hornbeak DM, Young TL: Myopia genetics: a review of current research and emerging trends. Curr Opin Ophthalmol 2009, 20:356-362

46. Ramrattan RS, van der Schaft TL, Mooy CM, de Bruijn WC, Mulder PG, de Jong PT: Morphometric analysis of Bruch's membrane, the choriocapillaris, and the choroid in aging. Invest Ophthalmol Vis Sci 1994, 35:2857-2864

47. Seddon JM, McLeod DS, Bhutto IA, Villalonga MB, Silver RE, Wenick AS, Edwards MM, Lutty GA: Histopathological insights into choroidal vascular loss in clinically documented cases of age-related macular degeneration. JAMA Ophthalmol 2016, 134: $1272-1280$

48. Whitmore SS, Braun TA, Skeie JM, Haas CM, Sohn EH, Stone EM, Scheetz TE, Mullins RF: Altered gene expression in dry age-related macular degeneration suggests early loss of choroidal endothelial cells. Mol Vis 2013, 19:2274-2297

49. Yuan X, Gu X, Crabb JS, Yue X, Shadrach K, Hollyfield JG, Crabb JW: Quantitative proteomics: comparison of the macular Bruch membrane/choroid complex from age-related macular degeneration and normal eyes. Mol Cell Proteomics 2010, 9:1031-1046

50. Bird AC, Phillips RL, Hageman GS: Geographic atrophy: a histopathological assessment. JAMA Ophthalmol 2014, 132:338-345

51. Li M, Huisingh $\mathrm{C}$, Messinger J, Dolz-Marco R, Ferrara D, Freund KB, Curcio CA: Histology of geographic atrophy secondary to age-related macular degeneration: a multilayer approach. Retina 2018, 38:1937-1953

52. Bhutto IA, McLeod DS, Jing T, Sunness JS, Seddon JM, Lutty GA: Increased choroidal mast cells and their degranulation in age-related macular degeneration. Br J Ophthalmol 2016, 100:720-726

53. Lutty GA, McLeod DS: Phosphatase enzyme histochemistry for studying vascular hierarchy, pathology, and endothelial cell dysfunction in retina and choroid. Vision Res 2005, 45:3504-3511

54. Wangsa-Wirawan ND, Linsenmeier RA: Retinal oxygen: fundamental and clinical aspects. Arch Ophthalmol 2003, 121:547-557

55. Songstad AE, Wiley LA, Duong K, Kaalberg E, Flamme-Wiese MJ, Cranston CM, Riker MJ, Levasseur D, Stone EM, Mullins RF, Tucker BA: Generating iPSC-derived choroidal endothelial cells to study age-related macular degeneration. Invest Ophthalmol Vis Sci 2015, 56:8258-8267 